\title{
Multi-Agent versus Multi-Robot and Other Byzantine Discussions
}

\author{
Vicente Matellán \\ Editor of the special issue on multi-robot systems \\ E-mail: vicente.matellan@unileon.es
}

\begin{abstract}
This special issue of the Journal of Physical Agents is devoted to multi-robot systems. Some might perhaps argue that the term should have been "multi-agent systems" or "physical multi-agent systems", in accordance with the title of the journal. In any language, there are clusters of several words with closely similar meanings. Expressions in science and technology are no exceptions, although they are supposed to be more specific. In particular, in the world of computer science, the term "agent" has become a buzzword and has to be combined with numerous adjectives to be correctly understood. This short introductory article will try to justify why the name was chosen for the journal, but not for this special issue, the intended scope of the journal and the contents of this issue.
\end{abstract}

Index Terms-JoPhA, agent, robot, distributed.

\section{JOPHA SCOPE}

$\mathbf{W}$ HEN a new journal is born, it has to define its own editorial line. When their promoters decide to establish it, they have a clear idea in their minds about its scope, and it is usual for all of them to think that this is well defined and even that their views coincide perfectly. However, it is impossible for all of them to share exactly the same idea about what it will look like. There will be shades of opinion due to their personal interests, education, environment, and so forth that will prevent any group of humans from achieving this perfect coincidence.

The evolution of a publication over time will create its own personality. In this process the founders are not the dominant factor. The authors sending in papers and, above all, the editors and reviewers will determine this by giving greater prominence to some papers than to others, in such a way defining the real scope of the journal.

The Journal of Physical Agents is no exception. In the scope description of this new journal the editors stated that it was seeking contributions in all areas that use agentbased technologies. In particular, mobile robotics, autonomous systems, or domotic systems were cited as among potential areas of interest for the journal.

This was the seminal idea of the founders of the journal, the members of the Spanish National Network on Physical Agents. In this grouping, we had been organizing national meetings for almost 10 years and realized we had not found a regular publication reflecting our common interests, at either a local or an international level.

Vicente Matellán is with the department of Mechanical and Computer Engineering, University de León. Edif. Tecnológico II (D-333). Campus de Vegazana. 24071 León (Spain).
Most of the members of this community work with robots. However, typically we are not robot builders, which makes us appear to fall in the category "soft-robotics" from the point of view of the traditional robotics community. In this sense, existing robotics journals do not fit our work well, although they are our most usual route for dissemination of research. In other words, we did not want to create yet another robotics journal.

Much of the work that we undertake does not use robots alone, although it can be considered as work in robotics. We are interested in any type of device that can be controlled by a computer, that is connected to the real world (the physical world, we mean), and that is able to establish communication with other devices. This might be our definition of a physical agent. Figure 1 outlines it as being at the crossroads of the areas of several different research communities, focusing primarily on the intersection between robots and agents.

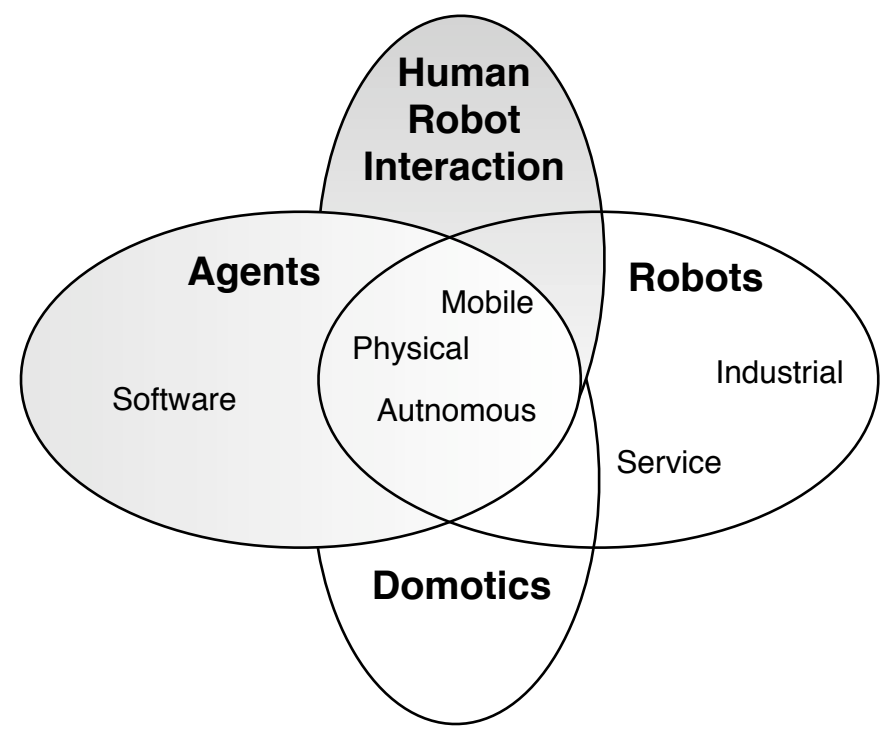

Fig. 1. JoPhA scope as seen by journal founders

As an example, in this issue, devoted to multi-robot systems, there are papers covering traditional autonomous legged robots, but also others on computerized cars. Similarly, the next issue will be given over to "Human interaction with domestic agents" , in other words, to an exploration of aspects of interaction with embodied devices such as Aibo, Pleo, Paro

\footnotetext{
${ }^{1}$ http://www.jopha.ua.es/JoPhA_CFP-.pdf
} 
and Nabaztag, or software such as Catz \& Dogz or Nintendogs, as well as the numerous non-commercial devices and systems that have been developed in many research labs. As was explained above, these numbers of the journal are defining its real scope for potential readers.

On the other hand, most people in this community are also involved in matters more closely related to "software agents", probably owing to their computer science background. They are also very active in that field, in both research and development. Such agents are not physical, but there have been many developments in this area that have influenced physical work. Moreover, physical agents can interact with software agents.

In addition it was realized that this profile is not merely a Spanish one, but can also be found in other countries. This encouraged us to establish the journal in English and give it an international scope. For instance, Dr. P. Stone, one of the authors of the articles that make up this issue, perfectly matches such a profile, having made significant contributions to the area of software agents, such as [12], but also being very active in traditional robotics, for instance [11].

Another question when a new journal is proposed is the desired level of applicability of the papers expected, in other words, whether it will be a theoretical or a practical journal. We think that in the current state of this field within computer science these two aspects can still be kept together, that there is not yet any formal corpus, so that many contributions are both theoretical and applied.

In short, JoPhA wishes to become a medium for the dissemination of theoretical and practical achievements in the field of physical agents, that is, in computer-controlled devices, embedded in the real world, capable of establishing communication with other similar devices and humans.

\section{A. Robot versus Agent}

The previous section attempted to focus on the scope of the journal. However, it should be stressed that from our point of view the expressions "robot" and "physical agent" can be used indistinguishably in some cases, but not all. In order to delimit these cases, an attempt will now be made to define the terms.

In the light of the above, "agent" and "robot" may have different meanings, depending on the author and the context. For instance, a reader coming from an industrial background is likely to see a robot as "A re-programmable multi-functional manipulator designed to move materials, parts, tools, or specialized devices through variable programmed motions for the performance of a variety of tasks" (Robotics Institute of America).

However, a reader from a computer science department would be more likely to define a robot as an autonomous, computer-controlled device made up of sensors and actuators. Something similar happens with the definition of an agent. The classic definition [14]describes an agent as a hardware or software-based computer system with the characteristics of autonomy, reactivity, pro-activeness and social ability.

Taking the first definition of robot, there would be few points of contact between the two areas. However, in robotics,

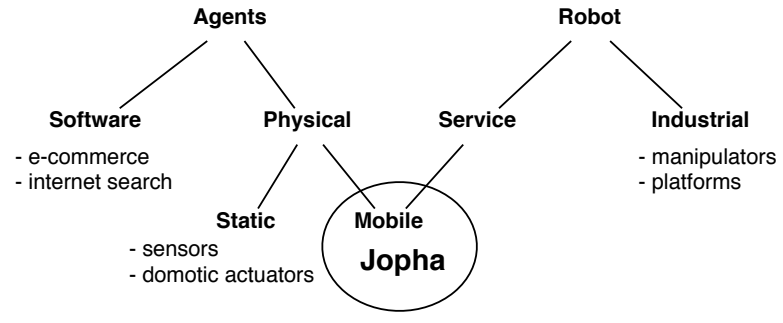

Fig. 2. Light taxonomy of robots and agents

as stated in [13], a mobile robot is often called a mobile agent, either virtual or physical. Nevertheless, sometimes a robot may be controlled by a single-agent architecture, while in other situations there may be several agents controlling the same robot.

Figure 2 tries to show the overlap between these two concepts. Robots can be divided into "industrial" robots, typically manipulators or automated platforms for in-factory delivery, and service robots, typically mobile robots. On the other hand, agents can be classified into software agents, usually Internet agents that can travel from one server to other, and physical agents, those that "live" in the real world. Hence, mobile robots and physical agents overlap.

This overlap has also appeared clearly in the literature. For instance, Dudek et al. [5] presents a detailed taxonomy of "multi-agent robotics", although this is not the only way of referring to multiple robot systems. For example, the expression "Cooperative robots" is used in [4], while in [2] "robot teams" is used. In brief, groups of mobile robots working together, or at least interacting, are among the clearest examples of physical agents that can be found.

\section{B. This issue content}

The contents of this issue are intended to show what we want the journal to be, in this instance focusing on the area of multi-robot systems. Hence, the issue includes various theoretical papers, such as the contribution by Bruno Lacerda et al. [9], alongside others that are much more applied, for example the piece by Patrick Beeson et al. [3]. An overview of the field is also included, in the shape of the invited paper by Lynne Parker [10], and a more applied contribution from Y. Elmaliach et al. [6] , emphasizing the problem of human-robot interaction in this domain.

\section{JOPHA FUTURE}

Our goals as a community at this point are simply to consolidate this publication as a quarterly journal ${ }^{2}$. We do not think that issues will be very large. We assume that an average issue will comprise 5 papers. On the other hand, we do not want to set maximum limits on the number of pages in a paper. If an author does need more space we are willing to offer it, obviously on condition that the relevant editor is in agreement.

\footnotetext{
${ }^{2}$ Note that 2008 will be the first complete year, and may be we just reach three issues
} 
We are making a bid to distribute the contents of the journal as libre (free) material, as well as to avoid any cost for authors. All of the contents are intended to become available on the Internet under a Creative Commons licence. However, any publishing effort of this kind needs some financial support. We would encourage all potential readers to subscribe themselves, or to ask their departments, universities, schools, or libraries to subscribe.

Finally, this is not an Internet or virtual journal. We prefer it to be a paper-based journal and wish to keep it in print form for many reasons. This is the traditional method for the dissemination of research, which we favour. Paper journals are very convenient, as they can be read anywhere (even in the absence of a net connection, or power), and libraries are a well-established and reliable method for storing and indexing knowledge.

\section{ACKNOWLEDGMENT}

I should like to recognize explicitly the great efforts made by all of the organizers of the annual sessions of the Spanish WAF (Workshop on Physical Agents), which have helped to create a local community around the "Physical Agent" concept in Spain and have given birth to this journal. In particular, I should like to express my extreme gratitude to Miguel A. Cazorla for his support and tireless work.

\section{REFERENCES}

[1] T. Arai, E. Pagello, and L.E. Parker, Advances in multi-robot systems. IEEE Transactions on Robotics and Automation, Vol. 18(5), pp. 655-661, 2002.
[2] Tucker Balch, and Lynne Parker, Robot Teams: From Diversity to Polymorphism. A.K. Peters, 2002.

[3] Patrick Beeson, Jack O'Quin, Bartley Gillan, Tarun Nimmagadda, Mickey Ristroph, David Li, and Peter Stone. Multiagent Interactions in Urban Driving. JoPhA Vol 2(1), 2008.

[4] Y. Uny Cao and Alex S. Fukunaga and Andrew B. Kahng. Cooperative Mobile Robotics: Antecedents and Directions, Autonomous Robots, Vol 4 (1), pp. 7-23, 1997.

[5] G. Dudek and M. Jenkin and E. Milios and D. Wilkes. A Taxonomy for Multi-Agent Robotics, Autonomous Robots, Vol. 3(4), pp. 375-397, 1996.

[6] Yehuda Elmaliach and Gal A. Kaminka. Robust Multi-Robot Formations under Human Supervision and Control. JoPhA Vol 2(1), 2008.

[7] L. Iocchi, D. Nardi, and M. Salerno, Reactivity and Deliberation: a Survey on Multi-Robot Systems, Lecture Notes in ComputerScience, Vol. 2103, pp. 9-34, 2001

[8] Editors: Amir Shapiro and Gal A. Kaminka. Special Issue on MultiRobot Coverage, Search, and Exploration. Annals of Math and Artificial Intelligence (AMAI) 2008 (to appear).

[9] Bruno Lacerda, Pedro Lima. Time Temporal Logic Control of Discrete Event Models of Cooperative Robots. JoPhA Vol 2(1), 2008.

[10] Lynne E. Parker. Distributed Intelligence: Overview of the Field and its Application in Multi-Robot Systems. JoPhA Vol 2(1), 2008.

[11] M. Sridharan and Peter Stone. Structure Based Color Learning on a Mobile Robot under Changing Illumination. Autonomous Robots, Vol. 23(3). pp. 161-182, 2007.

[12] Michael P. Wellman, Amy Greenwald, and Peter Stone. Autonomous Bidding Agents: Strategies and Lessons from the Trading Agent Competition, MIT Press, 2007.

[13] Grzegorz Wieczerzak, and Krzysztof Kozkowski. Agents that Live in Robots: How are successful applications built?. Fourth International Workshop on Robot Motion and Control, pp. 97-102, Puszczykowo (Poland) 2004

[14] M. Wooldridge, and N.R. Jennings, Intelligent Agents: Theory and Practice, Knowledge Engineering Review, Vol. 10(2), pp. 115-152, 1995. 